\title{
Dynamic Modeling and Simulation of Control System for Pneumatic Servo Valve Using Piezoelectric Actuator
}

\author{
Mohsen Heydari Beni ${ }^{1}$, Hadi Homaei ${ }^{2}$, Ehsan Kiani ${ }^{3}$, Fatemeh Abbaspour ${ }^{4}$
}

${ }^{1}$ M.Sc Student, Department of Mechanical Engineering, Shahrekord University, Shahrekord, Iran

${ }^{2}$ Assistant Professor, Department of Mechanical Engineering, Shahrekord University, Shahrekord, Iran

${ }^{3}$ B.Sc student, Department of Mechanical Engineering, Shahrekord University, Shahrekord, Iran

${ }^{4}$ M.Sc Student, Department of Mechanical Engineering, Shahrekord University, Shahrekord, Iran

\begin{abstract}
In this study design and simulation of controller for piezoelectric pneumatic servo valve is done. The dynamic model of the valve using analytical approach and the working principle of the servo valve is analyzed. The pole placement method is used to design a controller using observed variables. The valve controls the situation and position of actuator. Then for the system, full order state observer and PID controller based on ITAE (Integral Time Absolute Error) criteria are investigated and the response of system with these controllers is shown and a comparison is done for different schemes of controller design and the best design is selected for the system.
\end{abstract}

Keywords: Pneumatic Servo Valve, Piezoelectric Actuator, Full order State Observer, ITAE. 


\section{Introduction}

Position control applications have typically used one of two actuator technologies. Hydraulic actuators have speed and force profiles compatible with many industrial processes but can present a number of workplace hazards to personnel. Electromagnetic actuators, on the other hand, are clean and reliable in their operation but often require a mechanical transmission, both to convert high speed and low torque to a more useful combination and to convert rotary motion to linear motion. While linear motors can overcome the need for a transmission, they can be expensive. Pneumatic actuators afford the opportunity to design a positioning system that may be directly coupled with a load like a hydraulic actuator; is clean and reliable like electric motors; and is inexpensive. In recent years, in order to improve the control performance, several methods, such as feedback linearization, observer design, adaptive control, singular perturbation and fuzzy control, have been investigated in order to design effective control for different industrial control systems, such as robots, motors and pneumatic servo systems and etc.

Recently, pneumatic servo systems have been applied in several systems, such as pneumatic robot systems, spherical glass molding machines, and a precise positioning stage to isolate vibration. In other words, pneumatic technology is applied widely in many fields of industrial production with its advantages as high transmission speed, non-pollution and so on. At present, pneumatic technology has become one of the key technologies in modern control. Because air has a number of advantages, including compressibility, high power ratio, and low heat generation, and also air is non-magnetic and is a clean energy source. Traditional aerodynamic control valve uses electromagnet electrical - mechanical conversion drive and makes the control signal convert to the mechanical displacement, then it promotes the valve spool to achieve the control of access. However, it has shortcomings, such as large volume, low power consumption, slow response and susceptible to electromagnetic interference. Typically, however, the response times of pneumatic components are relatively long, especially if compared with the typical ones for electric and hydrodynamic components. In general, this slowness means that the bandwidth obtainable in pneumatic servo-systems is relatively limited. Numerous researchers have sought to increase this value to the maximum by changes to the hardware and the development of evolved control algorithms.

Pneumatic servo system control methods have been studied since the 1950s [1, 2]. The performance of the system improved greatly as the pneumatic servo valve became commercially available in the late 1980s [3]. The pneumatic servo valve is the key element in the system. Its structure and parameters are important in order to achieve good performance [4-8]. Recently, the need for improved controllability of the pneumatic servo system has increased with the development of precision manufacturing. That is high-performance pneumatic servo valve are needed.

In recent years, piezoelectric actuator has been applied in production as a new converter, and it is the device that converts the vibration of piezoelectric crystal to form the movement or power which is required by the driven part [911]. These actuators are capable to reach the high resolution and speed of the positioning object and possess many attractive features such as a short response time, a large output power, a compact size, self braking and good controllability [12-14]. Most of piezoelectric actuators are single degree-of-freedom (DOF) devices: it means that actuators generate a onedimensional linear or rotational motion of the moving part [14, 15]. Only few multi-degree-offreedom actuators are developed that are capable to actuate in many directions [15-18]. Obviously, the best way is to use one-directional piezoelectric actuator that can move or rotate the body in any direction.

The displacement generated by the piezoelectric crystal has a good linear relationship with the input signal. It has the advantages such as 
convenient to control, producing high driving forces, low power consumption and fast response. The inversing piezoelectric devices of piezoelectric materials replace the torque motor as the pre-amplifier of servo-electric components which can be achieved the fast, reliable, highprecision control of output signal [19].

In this report, the situation and position is controlled by piezoelectric pneumatic servo valve. In other words for this purpose, a controller is modeled and designed based on working principle of piezoelectric actuator. The method which is used for controller design is pole placement method. Full order state observer, controller of type PID based on ITAE performance index are investigated. ITAE is a performance index that is a quantitative measure of the performance of a system. Then response of system with these controllers is shown in some figures. For each design the characteristics of system that include percent overshoot, the rise time and settling time are compared together and finally the best design is selected for the system..

\section{Mathematical model of piezoelectric pneumatic servo valve}

Figure 1 shows the schematic diagram of movement mechanism of a lever-type spool valve. Referring to the structure, the piezoelectric stacked heap of laminated piezoelectric pneumatic servo valve is placed in the top of the valve body and parallel to the direction of spool valve movement, so it reduces the length of the valve and the structure becomes more compact.

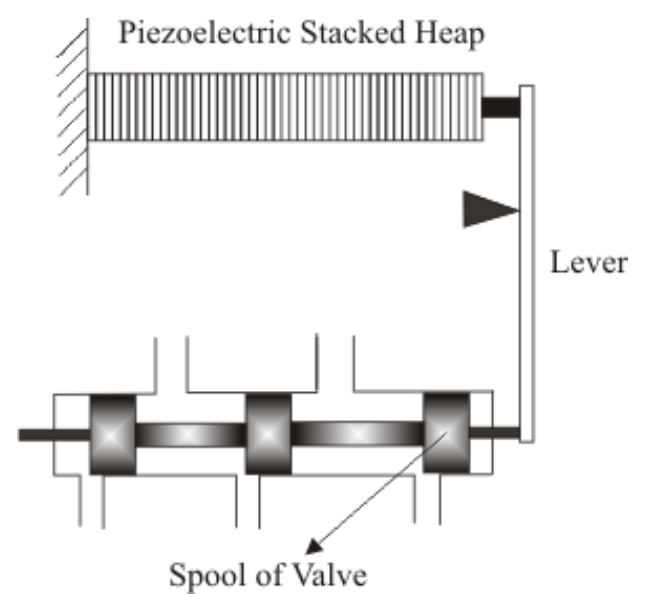

Figure 1: Piezoelectric pneumatic valve motion mechanism
According to figure 1, figure 2 shows the simplified dynamic model of spool movement mechanism.

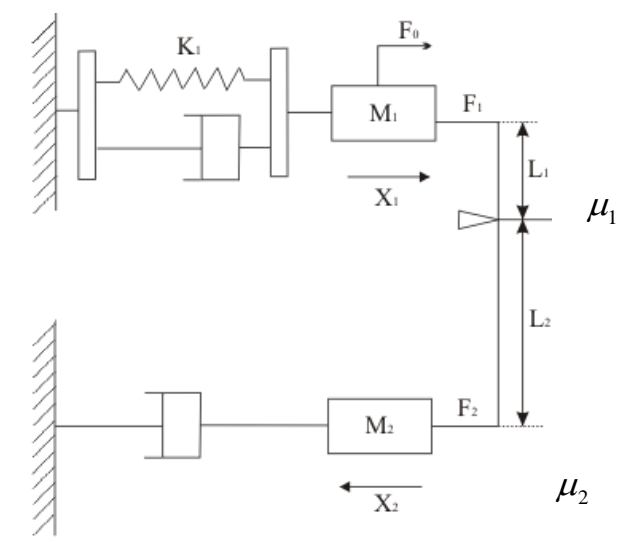

Figure 2: Dynamic model of spool motion mechanism

From this model, we can get out the differential equations of motion of the mechanism by Newton's second law:

$\left\{\begin{array}{l}m_{1} \ddot{x}_{1}+\mu_{1} \dot{x}_{1}+k_{1} x_{1}=F_{0}-F_{1} \\ F_{1} l_{1}-F_{2} l_{2}-I \ddot{\theta}=0 \\ m_{2} \ddot{x}_{2}+\mu_{2} \dot{x}_{2}=F_{2}\end{array}\right.$

Where $\mathrm{m}_{1}$ and $\mathrm{m}_{2}$ are equivalent mass of piezoelectric stack heap and spool, respectively. $\mu_{1}$ and $\mu_{2}$ are coefficients of damping, $F_{1}$ and $F_{2}$ are the force imposed from lever input or output and $\mathrm{F}_{0}$ is the transduced force from electrical domain (piezoelectric stack heap). $\mathrm{k}_{1}$ and I are the stiffness of the piezoelectric stack heap and movement inertia of amplification mechanism. It has been shown that a piezoelectric actuator can be modeled as a force generator $\mathrm{F}_{0}$ and stiffness $\mathrm{k}_{1}$. The generated force is:

$F_{0}=d_{33} n k_{1} v$

Where $d_{33}$ is the piezoelectric charge constant, $n$ is the number of layers, $v$ is the applied voltage. According to the servo valve differential equation list out the equation of input voltage $\mathrm{v}$ and spool displacement $x$ as: $\left(m_{1} \frac{l_{1}^{2}}{l_{2}}+m_{2} l_{2}+\frac{I}{b}\right) \ddot{x}_{2}+\mu_{2} l_{2} \dot{x}_{2}+2 k_{1} \frac{l_{1}^{2}}{l_{2}} x_{2}=k_{1} d_{33} n v l_{1}$

By doing Laplace transform to the equation (3), get out the transfer function as: 
$G_{0}(s)=\frac{x(s)}{v(s)}=\frac{b_{0}}{a_{2} s^{2}+a_{1} s+a_{0}}$

Where:

$B_{0}=\frac{b_{0}}{a_{2}} \quad \& \quad A_{0}=\frac{a_{0}}{a_{2}} \quad \& \quad A_{1}=\frac{a_{1}+a_{0} R C}{a_{2}} \quad \&$

$A_{2}=1+\frac{a_{1}}{a_{2}} \quad \& \quad A_{3}=R C$

The transfer function of piezoelectric pile is as follows:

$$
G_{1}(s)=\frac{v(s)}{v_{0}(s)}=\frac{1}{R C s+1}
$$

Where $v_{0}$ is the input voltage. Then the transfer function of the system will be:

$$
G(s)=G_{1}(s) G_{0}(s)=\frac{x(s)}{v_{0}(s)}=\frac{B_{0}}{A_{3} s^{3}+A_{2} s^{2}+A_{1} s+A_{0}}
$$

Where:

$$
\begin{aligned}
& B_{0}=\frac{b_{0}}{a_{2}} \& \quad A_{0}=\frac{a_{0}}{a_{2}} \& \quad A_{1}=\frac{a_{1}+a_{0} R C}{a_{2}} \quad \& \\
& A_{2}=1+\frac{a_{1}}{a_{2}} \& \quad A_{3}=R C
\end{aligned}
$$

The valve cylinder circuit is shown in Fig.3.

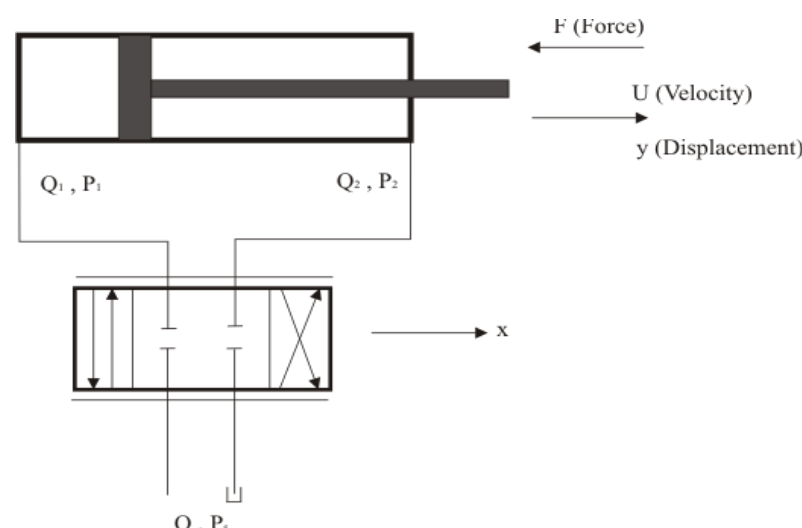

Figure 3: Valve Cylinder Circuit

For the simple system assume that the moving components have negligible inertia so that, as a consequence, the piston pressures will remain constant during transient changes caused by displacement of the valve. Thus:

$Q=k_{Q} x$

Where:
$k_{Q}=C_{Q} \pi d \sqrt{\frac{P_{s}}{\rho}} \quad m^{2} / s$

And $P_{s}$ is supply pressure, $d$ is valve spool

diameter, $\mathrm{C}_{Q}$ is flow coefficient and $\mathrm{Q}$ is the fluid density. For a piston area A, the piston velocity, $\mathrm{V}$ is as follow:

$V=\frac{Q}{A}=k_{Q} \frac{x}{A}$

As $V=\frac{d y}{d t}$, then $y=\frac{k_{Q}}{A} \int x d t$. The load force will affect the flow coefficient $k_{Q}$ in equation (7) because the valve pressure differences become:

$P_{s}-P_{1}=\frac{1}{2}\left(P_{s}-P_{m}\right)=P_{2}$

Equation (7) can be rewritten as:

$Q=C_{Q} \pi d x \sqrt{\left(\frac{P_{s}-P_{m}}{\rho}\right)}=k_{Q} x \sqrt{\left(1-\frac{P_{m}}{P_{s}}\right)}$

For $P_{m}=0$ (i.e zero force) equation (11) reverts to equation (7).

The compressibility of fluids is expressed by bulk modulus, $\beta$, which varies with pressure and as a consequence, either average or local values have to be used. Bulk modulus is defined as:

$$
\beta=-\frac{P}{\Delta V / V}
$$

Where the term $\frac{\Delta V}{V}$ is the volumetric strain in the fluid. Finally the transfer function between $\mathrm{V}$ and $Q$ is of the form:

$\frac{V}{Q}=\frac{C_{x} / A}{k_{Q}\left(1+2 \frac{\zeta}{\omega_{n}} s+\frac{1}{\omega_{n}^{2}} s^{2}\right)}$

Where, $\mathrm{V}$ is the Velocity of piston and

$\omega_{n}=\sqrt{\frac{2 \beta A^{2}}{V_{c} m_{p}}} \quad, \quad \zeta=\frac{C_{p}}{2 A} \sqrt{\frac{m_{p} \beta}{2 V_{c}}}+\frac{C_{f}}{2 A} \sqrt{\frac{V_{c}}{2 m_{p} \beta}}$

$C_{x}, C_{p}$ are flow coefficients, $C_{f}$ is viscous friction, $m_{p}$ is mass of piston, $\mathrm{V}_{\mathrm{c}}$ is volume of 
cylinder and $\mathrm{A}$ is piston area. Friction is usually considered to compose of coulomb, which is assumed to be unchanged, and a speed dependent element that is assumed to vary proportionally with the velocity. Changes in piston displacement are the integral of velocity that can be written as $y=\frac{V}{S}$. Thus, from equation (13) we get:

$$
\frac{y}{Q}=\frac{C_{x} / A}{k_{Q} s\left(1+2 \frac{\zeta}{\omega_{n}} s+\frac{1}{\omega_{n}^{2}} s^{2}\right)}
$$

Equation (15) relates flow rate of fluid to changes in the valve position with a constant external force acting on the piston rod.

Therefore the final transfer function of the system will be given as below:

$$
\frac{y}{v_{0}}=\frac{B_{0} C_{x} / A}{k_{Q} s\left(A_{3} s^{3}+A_{2} s^{2}+A_{1} s+A_{0}\right)\left(1+2 \frac{\zeta}{\omega_{n}} s+\frac{1}{\omega_{n}{ }^{2}} s^{2}\right)}
$$

\section{Controller Design}

3.1 Full-order state observer controller design :

The state observer estimates the state variables using the output and control input value. At this time, it can be configured only when the system is observable. $\widetilde{\mathbf{x}}$ is the observed state vector. Let's consider the system that is defined in form of a state-space representation as follows:

$$
\left\{\begin{array}{l}
\dot{\mathbf{x}}=\mathbf{A} \mathbf{x}+\mathbf{B u} \\
\mathbf{y}=\mathbf{C x}
\end{array}\right.
$$

Where $\mathbf{x}$, is called the state vector, $\dot{\mathbf{x}}$ the derivative of the state vector with respect to time, $\mathbf{y}$ the output vector and $\mathbf{u}(t)$ the input or control vector. Also $\mathbf{A}$ is the system matrix $(n \times n)$, B the input matrix $(n \times r), \mathbf{C}$ the output matrix $(m \times n)$ Suppose that $\tilde{\mathbf{x}}$ is an estimation for the state vector $\mathbf{x}$. Then, we have:

$$
\dot{\widetilde{\mathbf{x}}}=\mathbf{A} \tilde{\mathbf{x}}+\mathbf{B u}+\mathbf{K}_{\mathbf{e}}(\mathbf{y}-\mathbf{C} \tilde{\mathbf{x}})
$$

The equation (18) indicates the state observer. $\mathbf{y}$ and $\mathbf{u}$ are inputs and $\tilde{\mathbf{x}}$ is output. The term at the right end of the Eq.(18) corrects the difference between the measured output $\mathbf{y}$ and the estimated output $\tilde{\mathbf{x}} \cdot \mathbf{K}_{\mathrm{e}}$ works as the weighting matrix. Even though there is a difference between the matrix $\mathbf{A}$ and $\mathbf{B}$ of the actual system and the model, the influence is reduced by this correction. Deduct the equation (18) from the equation (17) to get the error of the observer. Then,

$$
\dot{\mathbf{x}}-\dot{\tilde{\mathbf{x}}}=\left(\mathbf{A}-\mathbf{K}_{\mathbf{e}} \mathbf{C}\right)(\mathbf{x}-\tilde{\mathbf{x}})
$$

The result is the equation (19). Suppose that the observer error $(\mathbf{x}-\tilde{\mathbf{x}})$ is e, that is to say, $\mathbf{e}=(\mathbf{x}-\tilde{\mathbf{x}})$, the equation (20) is arranged like the equation (20).

$\dot{\mathbf{e}}=\left(\mathbf{A}-\mathbf{K}_{\mathbf{e}} \mathbf{C}\right) \mathbf{e}$

If the given system is completely observable, we can make the eigenvalues of $\mathbf{A}-\mathbf{K}_{\mathbf{e}} \mathbf{C}$ as we want as selecting $\mathbf{K}_{\mathbf{e}}$ well.

In this study, for desired and expected conditions of design, the percent overshoot (\%OS) and the settling time $\left(\mathrm{T}_{\mathrm{s}}\right)$ are considered as the characteristic parameters.

Suppose that the transfer function for second order system is written as follows:

$G(s)=\frac{\omega_{n}^{2}}{s^{2}+2 \zeta \omega_{n} s+\omega_{n}^{2}}$

By using Eqs. (22) and (23), for second order system (Eq. (21)), $\zeta$ and $\omega_{n}$ are obtained as follows:

$\zeta=\frac{-\ln (\% O S / 100)}{\sqrt{\pi^{2}+(\ln (\% O S / 100))^{2}}}$

$\omega_{n}=\frac{4}{\zeta T_{s}}$

Then having $\zeta$ and $\omega_{n}$, the location of desired poles should be determined so that transient response is acceptable and rational. It is apparent that when the rank of the state observer matrix is equal to the order of transfer function of system, then the system is completely observable. The state observer matrix is as follows:

$\mathbf{N}=\left[\begin{array}{llll}\mathbf{C} & \mathbf{C A} & \ldots & \mathbf{C A}^{n-1}\end{array}\right]^{T}$

The transfer function of the full order controller observer is: 


$$
\frac{U(s)}{-Y(s)}=\mathbf{K}\left(s \mathbf{I}-\mathbf{A}+\mathbf{K}_{\mathbf{e}} \mathbf{C}+\mathbf{B K}\right)^{-1} \mathbf{K}_{\mathbf{e}}
$$

The state feedback gain matrix $K$ for this case is obtained by Ackerman's formula as follows:

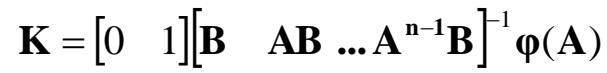

Where

$$
\boldsymbol{\varphi}(\mathbf{A})=\mathbf{A}^{n}+\alpha_{1} \mathbf{A}^{n-1}+\ldots+\alpha_{n} \mathbf{I}
$$

The desired characteristic polynomial is:

$$
\left(s+v_{1}\right)\left(s+v_{2}\right) \ldots\left(s+v_{n}\right)=|s \mathbf{I}-(\mathbf{A}-\mathbf{B K})|=\boldsymbol{\varphi}(s)
$$

Where $v$ 's are the desired egienvalues of the observer. The coefficients of function $\boldsymbol{\varphi}(s)$ are equal to $\alpha \mathrm{s}$ in the $\boldsymbol{\varphi ( A )}$.

As a general approximation rule, the observer poles must be two to five times faster than the controller poles to make sure the observation error converges to zero quickly. Having these poles and applying Ackermann's equation, the state observer gain matrix $\mathbf{K}_{\mathrm{e}}$ is given by:

$$
\mathbf{K}_{e}=\boldsymbol{\varphi}^{\prime}(\mathbf{A})\left[\begin{array}{llll}
\mathbf{C} & \mathbf{C A} & \ldots . & \mathbf{C A}^{n-1}
\end{array}\right]^{T}\left[\begin{array}{lllll}
0 & 0 & \ldots & 0 & 1
\end{array}\right]^{T}
$$

Where $\varphi^{\prime}(\mathbf{A})$ is the characteristic equation for desired poles of state observer. Then the transfer function of state observer is gained and the response of system to the initial condition can be derived.

\subsection{ITAE Performance Index:}

For any system, the required system performance can be specified quantitatively. Then it is important that a performance index can be calculated or measured and used to evaluate the system's performance. The best system is defined as the system that minimizes this index. This index is always positive or zero. To reduce the contribution of the large initial error to the value of the performance integral, as well as to emphasize errors occurring later in the response, the following index has been proposed:

$$
\text { ITAE }=\int_{0}^{T} t|e(t)| d t
$$

This performance index is designated the integral of time multiplied by absolute error and for optimum design, the system is chosen that its index is the least.
The PID controller has the transfer function as follows:

$G_{c}(s)=K_{P}+\frac{K_{I}}{s}+K_{D} s$

To implement such a controller, three parameters must be determined for the given process: proportional gain, integral gain, and derivative gain.

Consider the PID controller as:

$$
\begin{aligned}
& G_{c}(s)=K_{P}+\frac{K_{I}}{s}+K_{D} s=\frac{K_{D} s^{2}+K_{P} s+K_{I}}{s}= \\
& \frac{K_{D}\left(s^{2}+a s+b\right)}{s}=\frac{K_{D}\left(s+z_{1}\right)\left(s+z_{2}\right)}{s}
\end{aligned}
$$

where $a=K_{P} / K_{D}$ and $b=K_{I} / K_{D}$.

Therefore, a PID controller introduces a transfer function with one pole at the origin and two zeros

In general, we note that PID controllers are particularly useful for reducing steady-state error and improving the transient response when $\mathrm{Gc}(\mathrm{s})$ has one or two poles.

Hence, we select the three PID coefficients to minimize the ITAE performance index, which produces an excellent transient response to a step.

The optimum coefficients of the characteristic equation for ITAE are obtained from Eq. (33)

$$
s^{3}+1.75 \omega_{n} s^{2}+2.15 \omega_{n}{ }^{2} s+\omega_{n}{ }^{3}
$$

\section{Simulation and Results}

Based on the transfer function of the system, according to the pneumatic servo valve dynamic model and material properties, the parameters of the model can be considered as below:

$\mathrm{K}_{1}=2.5 \times 10^{7}(\mathrm{~N} / \mathrm{m}), \mathrm{L}_{1}=5 \mathrm{~mm}, \mathrm{~L}_{2}=40 \mathrm{~mm}, \mathrm{~m}_{1}=0.04 \mathrm{~kg}$, $\mathrm{m}_{2}=0.02 \mathrm{~kg}, \quad \mathrm{I}=5.4 \times 10^{-6}\left(\mathrm{~kg} / \mathrm{m}^{2}\right), \quad \mathrm{c}=3.6 \times 10^{-6}(\mathrm{~F})$, $\mathrm{R}=50 \Omega, \quad \mathrm{d}_{33}=1.5 \times 10^{-9}(\mathrm{~m} / \mathrm{V}), \quad \mu_{2}=1000 \mathrm{~N} /(\mathrm{m} / \mathrm{s})$, $\mathrm{Q}=7.5 \times 10^{-6}\left(\mathrm{~kg} / \mathrm{mm}^{3}\right), \mathrm{n}=500$

Also for the fluid:

$C_{Q}=0.65, \rho=870, \mathrm{dp}=50(\mathrm{~mm}), \mathrm{d}=$ $12(\mathrm{~mm}), k_{Q}=0.0012\left(\mathrm{~m}^{3} / \mathrm{s}\right) / \mathrm{m}, \beta=1.4^{*} 10^{9}\left(\mathrm{~N} / \mathrm{m}^{2}\right)$, $m_{p}=250(\mathrm{~kg}), \mathrm{V}=0.15^{*} 10^{-3}\left(\mathrm{~m}^{3}\right), \mathrm{A}=10^{-3}\left(\mathrm{~m}^{2}\right), \omega_{n}$ $=273(\mathrm{rad} / \mathrm{s})$,

$C_{p}=7.42^{*} 10^{-12} \quad\left(\mathrm{~m}^{3} / \mathrm{s}\right) /\left(\mathrm{N} / \mathrm{m}^{2}\right), C_{f}=16^{*} 10^{3}$

$\mathrm{N} /(\mathrm{m} / \mathrm{s}), C_{x}=0.96\left(\mathrm{~m}^{2} / \mathrm{s}\right), \zeta=0.243$ 
Using these quantities and discussed equations in previous section, the transfer function of system is obtained as follows:

$\frac{y}{V_{0}}=\frac{\operatorname{Num}(s)}{\operatorname{Den}(s)}$

Where:

$$
\operatorname{Num}(s)=1.3
$$

$\operatorname{Den}(s)=s^{6}+2.7 s^{5}+36.2 s^{4}+20.1 s^{3}+50.9 s^{2}+1.5 s$

\subsection{Design of full-order state observer}

We would like to design the full order observer controller such that in the unit step response the maximum overshoot is less than $10 \%$ and settling time is about $0.5 \mathrm{sec}$. We first design the controller by finding the desired characteristic equation.

A $10 \%$ overshoot and a settling time of 0.5 second yield $\xi=0.591$ and $\omega_{n}=13.53$ thus, the characteristic equation for dominant poles is $s^{2}+16 s+183.1=0$, where the dominant poles are located at $-8 \pm j 10.91$. Hence, choose the desired closed-loop poles at $s=\mu_{i}(\mathrm{i}=1,2)$ where $\mu_{1}=-8+j 10.91, \mu_{2}=-8-j 10.91$.

Also we choose the desired observer poles at $v_{1}=-35, v_{2}=-38$

For designing of full-order state observer, according to described method in previous section, using Ackerman equation (Eq. (24)) , the gain matrix $\mathrm{K}$ is considered as below:

$K_{e}=[-15625-18748.43-9324.02-2479.85-338.8-27.3]$

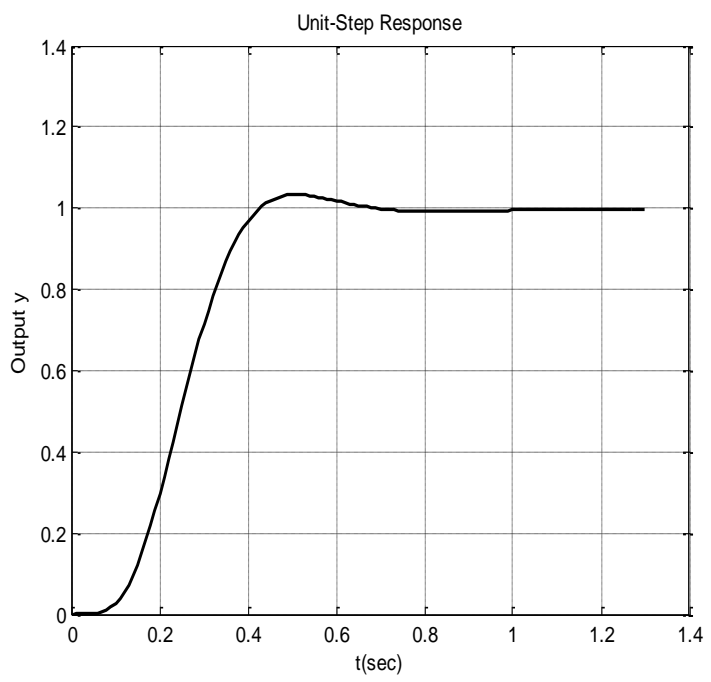

Figure 4: Response to unit step for full-order state observer

Also the transfer function of control system is acquired.

$\frac{U(s)}{-Y(s)}=\frac{-2.68 \mathrm{~s}^{5}-1 \mathrm{~s}^{4}-1.07 \mathrm{~s}^{3}-1.56 \mathrm{~s}^{2}-1.97 \mathrm{~s}+3.13}{\mathrm{~s}^{6}-2.04 \mathrm{~s}^{5}-3 \mathrm{~s}^{4}-1.71 \mathrm{~s}^{3}-4.96 \mathrm{~s}^{2}-8 \mathrm{~s}-7.41}$

Having the gain matrix of observer $\mathrm{K}_{\mathrm{e}}$ and transfer function of controller, the response to initial conditions can be drawn as in Fig. 5 .

\subsection{Design of controller type PID}

We would like to design the PID controller such that in the unit step response the maximum overshoot is less than $10 \%$ and settling time is about $0.5 \mathrm{sec}$.

In order to meet the settling time requirement, $\omega_{\mathrm{n}}$ is selected. Since Ts $=4 /\left(\xi \omega_{\mathrm{n}}\right)$ and $\xi$ is near 0.8 , and $\omega_{n}=10$. Then three coefficients are obtained from Eq. (33) as $K_{P}=214, K_{D}=15.5$ and $K_{I}=1000$. Step response for controller type PID is drawn in Fig. 6. It is seen that the percent overshoot is 30.4 and the steeling time is 77.4 second. 


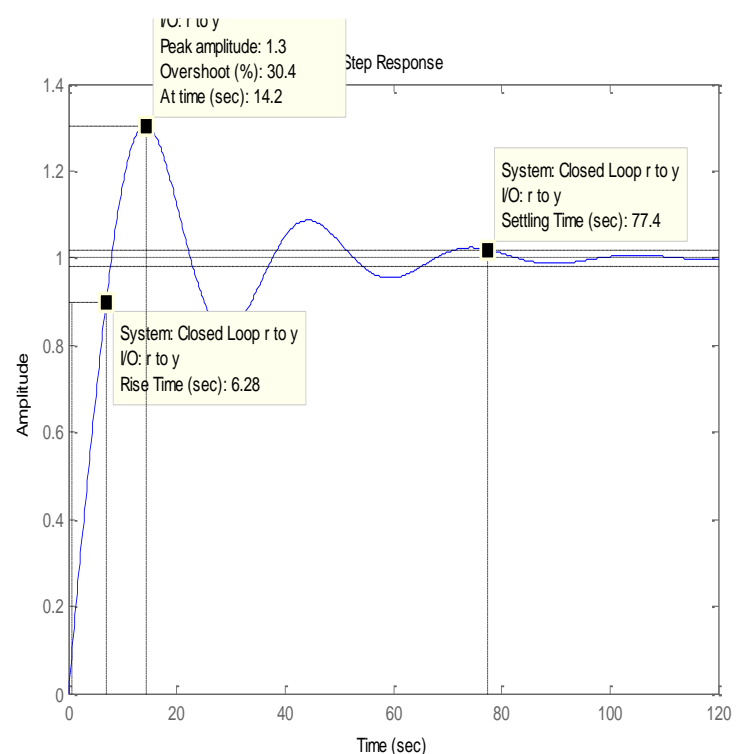

Figure 5: Step response for controller type PID

Table 1 presents percent overshoot, rise time and settling time of system for various type of controller.

Tabela 1: Characteristics of system for various types of controller

\begin{tabular}{cccc}
\hline $\begin{array}{c}\text { Various } \\
\text { type of } \\
\text { controller }\end{array}$ & $\begin{array}{c}\text { Percent } \\
\text { Overshoot }\end{array}$ & $\begin{array}{c}\text { Rise Time } \\
(\mathrm{s})\end{array}$ & $\begin{array}{c}\text { Settling } \\
\text { Time }(\mathrm{s})\end{array}$ \\
\hline $\begin{array}{c}\text { Full order } \\
\text { state } \\
\text { observer } \\
\text { PID }\end{array}$ & 4.5 & 0.3 & 0.7 \\
\hline
\end{tabular}

Comparing the results of design of full order state observer controller and PID controller, it is obvious that the best controller for the system is full order state observer. Because this type of controller presents the better performance than the PID controller.

\section{Conclusion}

In present study, the displacement of pneumatic actuator is controlled by piezoelectric pneumatic servo valve. At first piezoelectric pneumatic valve is modeled and then its differential equation of motion is derived. Then for the system, full order state observer and controllers of type PID based on ITAE criteria are designed. For these controllers the characteristics of system that include percent overshoot, the rise time and settling time, are compared together.
From this study, we can see that full order observer controller is more suitable for this application than the other controllers.

\section{References}

Andersen BW. (1967). The analysis design of pneumatic systems. John Wiley \& Sons, Inc.;.

BackeW. (1986). The application of servo pneumatic drives for flexible mechanical handling techniques. Robotics;2(1):45-56.

Burrows CR,Webb CR. (1966). Use of the root loci in design of pneumatic servomotors. Control;423-7.

Er Shi Ju. Research of piezoelectric-type servo valve. PhD thesis of Jilin University.

Festo, http://www.festo.com/INetDomino/coorp sites/en/index.htm

K. Uchino, (1997). Piezoelectric Actuators and Ultrasonic Motors, Kluwer Academic Publishers, Boston, Dordrech, London.

Krivts IL. (2004). Optimization of performance characteristics of electro-pneumatic two-stage servo valve. Trans ASME J Dyn Syst Measure Contr;126:416-20.

Liu S, Bobrow JE. (1988). An analysis of a pneumatic servo system and its application to a computer-controlled robot. Trans ASME Ser G: J Dyn Syst Measure Contr ;110:228-35.

M. Aoyagi, S.P. Beeby, N.M. White, (2002). A novel multi-degree-of-freedom thick-film ultrasonic motor, IEEE Transactions on Ultrasonics, Ferroelectrics and Frequency Control 49 (2) 151-158.

$\mathrm{Pu}$ J,Weston RH. (1989). Anewgeneration of pneumatic servo for industrial robot. Robotica ;7:17-23.

R. Bansevicius, R. Barauskas, G. Kulvietis, K. Ragulskis, (1988). Vibromotors for Precision Microrobots, Hemisphere Publishing Corp., USA.

S. Ueha, Y. Tomikawa, M. Kurosawa, K. Nakamura, (1993). Ultrasonic Motors: Theory and Applications, Clarendon Press, Oxford.

Shearer JL. (1956). Study of pneumatic processes in the continuous control of motion with compressed air-I, II. Trans ASME;233-49. 
T. Hemsel, J. Wallaschek, (2010). Survey of the present state of the art of piezoelectric linear motors, Ultrasonics 38 37-40.

Tao Xiang Ting, Yuan Rui Bo, Luo Jing. (2007). The application situation and development prospect of Pneumatic manipulator[ J]. Machine tools and hydraulic, 35(8):226-228.

Y. Gouda, K. Nakamura, S. Ueha, (2005). A miniaturization of the multi-degree-offreedom ultrasonic actuator using a small cylinder fixed on a substrate, in: Proceedings of the second International Workshop on Piezoelectric Materials and Applications in Actuators, Paderborn, Germany, pp. 263-267.

Zhang Hong Jia, WANG Ji Wei, Huang Yi. (2005). hydraulic and pneumatic[M].Beijing:China machine press.

Zuo Jian Ming. (2005). Hydraulic and Pneumatic Transmission[M]. Version 2.Beijing. Mechanical industry press. 